\title{
Integração do ensino, pesquisa e extensão universitária na formação acadêmica: percepção do discente de enfermagem
}

\author{
Integration of teaching, research and university extension in the academic formation: \\ perception of the student of nursing
}
Integración de la enseñanza, investigación y extensión universitaria en la formación académica: percepción del alumnado de enfermería

Daniela Paola Santos de Paula ${ }^{1}$, Mirielle Dias Gonçalves ${ }^{1}$, Marcos Gabriel de Jesus Rodrigues ${ }^{1}$, Rafhael Souza Pereira², Janaína Ribeiro Oliveira Fonseca ${ }^{3 *}$, Amanda Souto Machado ${ }^{3}$, Victor Hugo Dantas Guimarães ${ }^{4}$, João Marcus Oliveira Andrade ${ }^{5}$, Alanna Fernandes Paraíso ${ }^{5}$.

\section{RESUMO}

Objetivo: Compreender a percepção de acadêmicos do curso de graduação em Enfermagem sobre a integração entre ensino, pesquisa e extensão durante sua formação acadêmica. Métodos: Consiste em estudo transversal, de abordagem qualitativa, realizado com acadêmicos e egressos do curso de Enfermagem de uma instituição de Ensino Superior privada na cidade de Montes Claros, norte de Minas Gerais, Brasil. Participaram do estudo 14 indivíduos que atenderam aos critérios de inclusão. Após a coleta, os dados passaram por análise de conteúdo. O estudo foi autorizado pelo Comitê de Ética em Pesquisa sob o parecer 2.565.451/2018. Resultados: A partir da análise de dados foi possível traçar o perfil da amostra estudada e conhecer as percepções e experiências destes alunos em relação à pesquisa e à extensão. Conclusão: Observou-se que a integração do ensino, da pesquisa e da extensão universitária é de grande importância para a formação de profissionais capacitados, qualificados e que usam efetivamente a prática baseada em evidências.

Palavras-chave: Enfermagem, Pesquisa, Extensão Universitária.

\begin{abstract}
Objective: To understand the perception of nursing scholars about the integration of teaching, research, and extension during their university education development. Methodology: This is a cross-sectional and qualitative study carried out with nursing undergraduate and graduate students of an University in the North of Minas Gerais. 14 individuals met the inclusion criteria. The data was collected and submitted to content analysis. The study was approved by the Research Ethics Committee under the protocol number 2.565.451/2018. Results: from the data analysis it was possible to trace the student's profile and to understand their perceptions and experiences in the research and extension fields. Conclusion: It was observed that the integration of teaching, research, and university extension are of great importance to the training of skilled, and qualified professionals who effectively use evidence-based practice.
\end{abstract}

Key words: Keywords: Nursing, Search, University Extension.

\footnotetext{
${ }^{1}$ Faculdade de Saúde e Desenvolvimento Humano Santo Agostinho, Montes Claros - Minas Gerais.

${ }^{2}$ Faculdade de Ciências Exatas e Tecnológicas Santo Agostinho, Montes Claros - Minas Gerais.

3 Universidade Estadual de Montes Claros - Unimontes, Montes Claros - Minas Gerais.

${ }^{4}$ Universidade Estadual de Montes Claros - Unimontes, Montes Claros - Minas Gerais.

${ }^{5}$ Faculdade de Saúde e Desenvolvimento Humano Santo Agostinho, Montes Claros - Minas Gerais.

*E-mail: jana.mg@hotmail.com
} 


\section{RESUMEN}

Objetivo: Comprender la percepción de los académicos del curso de graduación en enfermería sobre la integración entre la enseñanza, la pesquisa y extensión durante su formación académica. Metodología: Consistente en un estudio transversal, de abordaje cualitativo realizado con académicos y graduados del curso de enfermería de una institución de enseñanza superior privada en Montes Claros, Minas Gerais, Brasil. Participaron del estudio 14 individuos que atendieron a los criterios de selección. Tras la recolección, los datos pasaron por análisis de contenido. El estudio fue autorizado por el Comité de Ética en Pesquisa autorización 2.565.451/2018. Resultados: A partir del análisis de los datos fue posible trazar el perfil de la muestra estudiada y conocer las percepciones y las experiencias de dichos alumnos con la pesquisa y extensión. Conclusión: Se observó que la integración de la enseñanza, la pesquisa y la extensión universitaria son de gran importancia para la formación de profesionales altamente calificados y que usen efectivamente la práctica basada en evidencias.

Palabras clave: Enfermería, Pesquisa, Extensión Universitaria.

\section{INTRODUÇÃO}

Conhecida por ser um espaço que possibilita a agregação de inúmeros saberes, a universidade é a base para a formação acadêmica, desenvolvimento profissional e social. De acordo com os pressupostos da Lei de Diretrizes e Bases da Educação Nacional (1996), a educação superior tem por finalidade estimular no acadêmico o conhecimento dos problemas do mundo, prestar serviços especializados à comunidade, estabelecendo com esta uma relação de reciprocidade (FERNANDES MC, et al., 2012; BRASIL, 1996).

A constituição Federal de 1988 aborda as atividades de pesquisa e extensão como um leque de possibilidades para a execução da autonomia didático-cientifica das Universidades, sempre obedecendo os princípios da indissociabilidade de seu eixo fundamental: ensino, pesquisa e extensão (DIVINO AEA, et al., 2013).

A Reforma Sanitarista e a criação do Sistema Único de Saúde (SUS), na década de 1980 e 1990, indicaram mudanças para os cursos de saúde, trazendo à tona um novo perfil epidemiológico, político e social (EDUARDO EA, 2016).

Para acompanhar as transformações decorrentes das constantes modificações no quadro político-social e econômico do Brasil, o ensino de graduação na área da saúde vem sofrendo sucessivas mudanças curriculares, a partir de amplas discussões de novas propostas pedagógicas, no intuito de formar profissionais que atendam às necessidades do mercado de trabalho e de saúde da população (FERNANDES MC, et al., 2012;BARLEM BGT, et al., 2012).

A tendência em adotar o modelo biomédico de ensino em oposição a visão multidisciplinar, gera um déficit na formação de enfermeiros e outros profissionais de saúde. Esse fato, distancia os graduandos das questões políticas, socioeconômicas e científicas relativas à sua profissão, sendo priorizado o conhecimento técnico apenas. Diante desse fato, os profissionais têm sido preparados para a resolução dos desafios tecnológicos, mas pouco sensíveis ao uso social da ciência (SIQUEIRA SMS, et al., 2017).

A integração entre o ensino, a pesquisa e a extensão transcendem as noções sistêmicas, técnicas e biologicistas. Diante desse novo cenário, a visão de profissional competente passa a ser baseada no conhecimento multidimensional, em que, por meio de um olhar crítico e clínico, o profissional consiga contextualizar, sistematizar e empreender o conhecimento possibilitando a transformação social (PIVETTA HMF, et al., 2010).

Piveta HMF et al. (2010) ainda afirmam que esta integração fomenta uma formação profissional que ultrapassa aspectos técnicos, instrumentais ou teóricos, trazendo, a partir de uma ampla visão, a fusão de aspectos técnico-científicos, éticos, políticos, econômicos e sócio-culturais. 
Desse modo, surge um ciclo inteligente onde a geração e aprimoramento dos conhecimentos, que são propagados através do ensino e extensão, fazendo com que haja uma integração efetiva entre os pilares da formação (DIVINO AEA, et al., 2013).

Visando a incrementação dos avanços emergentes na formação de profissionais de saúde, tem sido implementadas alterações contínuas nas matrizes curriculares dos cursos da área da saúde, entendendo que a estrutura curricular deve sempre ser adequadas as necessidades da formação de profissionais da saúde no Brasil (SILVA VO e SANTANA PMMAA,2015).

Entretanto, ainda existe uma deficiência na formação de enfermeiros e outros profissionais de saúde que relacionem as questões politicas socioeconômicas e cientificas, uma vez que, na maioria das vezes o conhecimento técnico é priorizado (SIQUEIRA SMC, et al. ,2017).

Surge então a necessidade de avaliar o conhecimento dos acadêmicos de Enfermagem a respeito da importância da integração entre ensino, pesquisa e extensão na formação de profissionais com competências interativas, associativas e socialmente responsáveis, que tenham capadidade de atuar frente à complexidade dos problemas sociais emergentes, na realidade social.

Desse modo, o presente estudo tem por objetivo compreender a percepção de acadêmicos de enfermagem sobre a integração entre ensino, pesquisa e extensão durante sua formação acadêmica.

\section{MÉTODOS}

Trata-se de estudo transversal de abordagem qualitativa, realizado no período de março a maio de 2018, com acadêmicos matriculados e egressos do curso de Enfermagem da instituição de Ensino Superior, Faculdades Santo Agostinho, localizada na cidade de Montes Claros, norte de Minas Gerais, Brasil (ARAGÃO J, 2011; MINAYO MCS, 2012; FASA, 2017; IBGE, 2017).

Buscando a construção de propostas que permitam a integração entre o serviço, o ensino e a comunidade de forma intrínseca à matriz curricular. A instituição, através da coordenação do curso de graduação em Enfermagem, vem implantando desde 2015, os projetos de extensão "Meu Curso em Minha Cidade" e "Assistência à saúde de povos e comunidades tradicionais indígenas e quilombolas", como respostas às tendências das Diretrizes Curriculares dos cursos da área da saúde, com o objetivo de fornecer subsídios em situações reais de trabalho para a prática do cuidado de Enfermagem (FASA, 2017).

Assim, foram incluídos no estudo, acadêmicos que participaram das atividades dos projetos de extensão "Meu Curso em Minha Cidade" e "Assistência à saúde de povos e comunidades tradicionais indígenas e quilombolas".

A seleção da amostra constituiu-se em duas etapas. No primeiro momento, realizou-se a inserção de todos os acadêmicos que participaram das atividades dos projetos no período de março de 2015 a dezembro de 2017, em um banco de dados do Excel, mediante dados contidos na apólice de seguro e lista de presença dos alunos. Obteve-se a relação de todas as edições dos projetos e dos acadêmicos participantes, totalizando 23 ações, sendo $03 \mathrm{em}$ aldeias indígenas, 02 quilombolas e $18 \mathrm{em}$ municípios situados na região norte e sudoeste do estado de Minas Gerais, com participação de 385 acadêmicos de vários cursos. Nesse momento, em virtude das características amostrais usadas neste estudo, foram excluídos acadêmicos de outros cursos de graduação, presença de duplicatas ou triplicatas (acadêmicos que participaram de 2 ações ou mais).

Em segundo momento, todos os nomes foram inseridos em um script elaborado através da linguagem de programação Hipertext Preprocessor (PHP) associada ao HTML que gerou um algoritmo que realizava uma seleção aleatória e sem repetições de 30 nomes. Esse sorteio foi realizado com o objetivo de garantir a fidedignidade do estudo e viés de seleção (SILVA MS, 2008; BENTO EJ, 2013).

Dos 30 selecionados, 16 participantes foram excluídos após 03 tentativas de encontro sem sucesso, ou por terem desistido do curso. Finalizou-se, assim, a amostra com 14 selecionados, acadêmicos e egressos do curso de graduação em Enfermagem da instituição. 
Para a coleta de dados, foi utilizado um roteiro de entrevista semiestruturado elaborado pelos próprios pesquisadores contendo duas etapas, a saber: Questionário sociodemográfico e Entrevista semiestruturada contendo 17 questões norteadoras que abordavam as percepções e experiências dos acadêmicos.

Para garantir a fidedignidade do estudo, as entrevistas foram realizadas de forma individualizada e com uso do aparelho gravador que assegurava a transcrição dos discursos na íntegra para posterior análise por saturação (FONTANELLA BJB, et al., 2008).

Após a coleta dos dados, estes foram plotados em banco de dados eletrônico, em que cada aluno foi identificado como AC acompanhado pelo número de ordem das entrevistas. Os dados sociodemográficos foram tabulados e feita uma análise descritiva dos dados e os discursos foram analisados através do uso critérios de saturação (BARDIN L, 2009).

$O$ desenvolvimento do estudo atendeu às normas nacionais e internacionais de ética em pesquisa envolvendo seres humanos, aprovado sob parecer de nำ. 565.451/2018 (BRASIL, 2013).

\section{RESULTADOS}

A partir da análise dos dados sociodemográficos fornecidos, foi possível traçar o perfil da amostra, baseado em critérios de definição do Ministério da Saúde e do Ministério do Trabalho e Emprego através das variáveis Sexo, Idade, Etnia, Ocupação e Período em Curso estratificados nas Tabelas 1 e 2 descritas abaixo (BRASIL, 2010; RODRIGUES ALL, et al., 2013).

Tabela 1 - Perfil Sociodemográfico dos discentes do curso de enfermagem, Montes Claros, Minas Gerais. Março/Maio, 2018.

\begin{tabular}{|c|c|c|c|c|}
\hline \multirow[t]{2}{*}{ Variável } & \multicolumn{2}{|c|}{ Gênero } & \multicolumn{2}{|c|}{ Geral } \\
\hline & \multirow{2}{*}{\multicolumn{2}{|c|}{ Feminino }} & $\mathbf{N}$ & $\%$ \\
\hline \multirow[t]{3}{*}{ Sexo } & & & 8 & 57,14 \\
\hline & \multicolumn{2}{|c|}{ Masculino } & 6 & 42,85 \\
\hline & \multicolumn{2}{|c|}{-} & Idade Média & Geral \\
\hline \multirow[t]{2}{*}{ Idade } & \multicolumn{2}{|c|}{ Feminino } & 21,87 & - \\
\hline & \multicolumn{2}{|c|}{ Masculino } & 31.16 & - \\
\hline Etnia & Feminino & Masculino & $\mathbf{N}$ & $\%$ \\
\hline Amarela & 1 & - & 1 & 7,14 \\
\hline Branca & 1 & - & 1 & 7,14 \\
\hline Negra & - & 1 & 1 & 7,14 \\
\hline Parda & 6 & 5 & 11 & 78,57 \\
\hline Total & & & 14 & $100 \%$ \\
\hline
\end{tabular}

Fonte: Dados da pesquisa, 2018.

Observou-se que, mesmo a amostra tendo sido definida aleatoriamente, houve um equilíbrio na estratificação de sexo $57,14 \%$ mulheres $(n=8)$ e $42,85 \%$ homens $(n=6)$. Além disso, notou-se que, quanto à idade média global, prevaleceu a estratificação de adultos jovens (aproximadamente 26 anos), quanto à etnia prevaleceu a classificação autorreferida $78,57 \%(n=11)$ de pardos.

Quanto às variáveis ocupação e período em curso, grande maioria dos indivíduos atuavam na área da saúde, como acadêmicos ou técnicos de enfermagem $35,71 \%(n=5)$. Na referida Instituição de Ensino, o curso possui duração de 04 anos, sendo dividido em oito períodos letivos. Desse modo, cerca de metade da amostra concentrava-se no último período da graduação.

Para melhor compreensão, os discursos foram plotados em duas categorias que abordam as percepções e experiências dos alunos quanto ao ensino, pesquisa e extensão universitária e as barreiras encontradas no caminho do pensamento crítico. 
Tabela 2 - Características da formação acadêmica dos discentes do curso de Enfermagem, Montes Claros, Minas Gerais. Março/Maio, 2018.

\begin{tabular}{|c|c|c|c|}
\hline Variável & & $\mathbf{N}$ & $\%$ \\
\hline \multirow[t]{5}{*}{ Ocupação } & Agentes e auxiliares administrativos & 1 & 7,14 \\
\hline & Outros & 2 & 14,28 \\
\hline & Operadores do comércio em lojas e mercados & 2 & 14,28 \\
\hline & Estudante de nível superior & 4 & 14,28 \\
\hline & Trabalhadores em serviço de promoção e apoio a saúde & ${ }^{5} \mathrm{~N}$ & $\begin{array}{l}35,71 \\
\%\end{array}$ \\
\hline \multirow[t]{5}{*}{ Período em curso } & Terceiro Período & 1 & 7,14 \\
\hline & Quinto Período & 4 & 28,57 \\
\hline & Sétimo Período & 1 & 7,14 \\
\hline & Oitavo Período & 7 & 50,0 \\
\hline & Egresso & 1 & 7,14 \\
\hline Total & & 14 & $100 \%$ \\
\hline
\end{tabular}

Fonte: Dados da pesquisa, 2018.

\section{Ensino, Pesquisa e Extensão Universitária: Percepções e Experiências.}

Ao realizar entrevista, os acadêmicos demonstraram a importância da pesquisa e extensão durante a formação acadêmica.

"(...)É de grande relevância para o acadêmico, porque, além de trazer uma perspectiva melhor pra experiências, pro seu próprio "eu", principalmente ter contato com as pessoas que você nem imaginava..." (Acad. 02).

“(...) É um início de uma nova carreira. Porque através da pesquisa, das extensões, das ações, a gente desenvolve um querer melhor, ter uma visão mais preliminar do futuro. No presente, o que a gente quer ser mesmo..." (Acad. 02).

"(...) tem um papel fundamental, pois assim o aluno e o acadêmico podem desenvolver as suas habilidades na área da pesquisa, uma área que, é sem dúvida nenhuma, de fundamental importância para o crescimento da profissão em si, pois é com as novas pesquisas e estudos que podemos desenvolver a nossa carreira acadêmica" (Acad. 10).

“(...) A prática universitária nos levou a demonstrar uma experiência muito grande durante uma determinada ação na comunidade, o que trouxe benefícios tanto pra nós na comunidade, tanto pra nós como acadêmicos, digo, quanto para a comunidade" (Acad. 11).

\section{Pesquisa e Extensão Universitária: As barreiras encontradas no caminho do pensamento crítico}

Nesta seção, entrevistados abordaram as dificuldades encontradas para inserção na pesquisa e extensão:

"(...) Como eu vim de transferência, na outra instituição não tinha e quando tinha não dava pra mim porque eu moro distante, Varzelândia” (Acad. 3).

"(...) A falta de divulgação muitas vezes dos eventos de extensão, e a falta de divulgação de alguns eventos científicos e falta de tempo (...) muita gente trabalha, as dificuldades que eu encontrei foram essas e acredito que muitas pessoas também" (Acad. 9)

“(...) A questão do tempo, do horário de serviço, a maioria das atividades de extensão e pesquisa é feito, no período da manhã e da tarde, isso dificulta para as pessoas que trabalham como é o meu caso" (Acad. 10). 
Os alunos também discorreram sobre a importância da pesquisa, extensão e inserção do acadêmico neste ambiente:

\begin{abstract}
"(...) Tem o grupo de pesquisa e extensão dentro da faculdade no qual você mandava currículo e era selecionado (...) assim pra quem já gostava era muito motivador porque fazia o que gostava pra quem já não tinha muito interesse como eu que tinha essa retração, era mais difícil inserir no grupo" (Acad. 13).
\end{abstract}

“(...) Participei de algumas reuniões do grupo de extensão, acompanhado pela professora A.P onde ela nos direcionou em relação à pesquisa, então o quanto é importante em uma formação acadêmica, quem tem intenção de seguir em uma das dimensões da Enfermagem, que é a pesquisa, é muito importante desde o princípio, lá quando começa com metodologia científica, você começa buscar esse conhecimento para poder agregar novos conhecimentos em relação à pesquisa" (Acad. 14).

(...) A minha visão é que a pesquisa universitária auxilia muito, mas ainda é pouco instituída, as universidades não têm uma abrangência total ainda da pesquisa no campo universitário, e isso dificulta um pouco, porque nem todos os acadêmicos têm acesso isso" (Acad. 9).

“(...) O contato dos universitários em relação à extensão, ele é muito gratificante. Uma vez que você põe em prática todo o seu conhecimento teórico e favorece o conhecimento tanto do acadêmico, tanto do... pra... em benefício da comunidade" (Acad. 11)

“(...) A extensão e pesquisa é de fundamental importância a nossa formação, pois ela abre os caminhos, sem ela o profissional não consegue se atualizar, e gabaritar o seu currículo profissional" (Acad. 10).

“(...) As ações promovidas pelo grupo de extensão e pesquisa, como meu curso minha cidade, ações em praças e grupos de discussão com a população onde foram debatidos os temas em questão proposto a sociedades, é de relevância, pois assim podemos mostrar para a sociedade como é a faculdade e podemos também, é, ajudálos, é, em uma maior obtenção do saber sobre tudo que é feito durante o período de formação" (Acad. 10).

\title{
DISCUSSÃO
}

\section{Ensino, Pesquisa e Extensão Universitária: Percepções e Experiências.}

Para os entrevistados, o ensino, pesquisa e extensão são de suma importância para a vida acadêmica e profissional, a maioria das falas aborda a importância da interação acadêmica com a comunidade. Acreditase que o perfil formativo, segundo a experiência delineada, é conquistado por meio da integração entre ensino, pesquisa e extensão universitária. A integração desse tripé permite ao acadêmico ser inserido nos diferentes cenários, sendo alicerce desde o primeiro período da graduação, não se tratando apenas de uma formação complementar, e sim proporcionando ao acadêmico visão diferenciada com fundamento nas perspectivas e benefícios (PIVETTA HMF, et al., 2010)

Aplicar na prática o conhecimento adquirido em sala de aula e desenvolver em ambiente externo proporciona contato entre o acadêmico e a sociedade a qual se torna beneficiada por meio do seu atendimento. Dessa forma, o acadêmico acaba aprendendo mais quando há esse contato, pois se torna gratificante praticar a teoria recebida dentro da sala de aula (RODRIGUES ALL, et al., 2013).

Essa relação entre a comunidade e instituições de Ensino Superior enriquece o processo de ensinoaprendizagem socializando o saber com a participação da comunidade na vida acadêmica. Esse elo entre os 
alunos, profissional e comunidade tornam-se fundamental para realimentar o ensino, trocar conhecimentos, experiências e para a prática da pesquisa científica (NEVES DS e MALTA SCL,2014). Nesse contexto, a extensão universitária surge como um processo educativo e científico, que articula o ensino e a pesquisa de forma indissociável, possibilitando uma relação entre a instituição de nível superior e a sociedade (RODRIGUES ALL, et al., 2013).

\section{Pesquisa e Extensão Universitária: As barreiras encontradas no caminho do pensamento crítico}

Para os entrevistados, as dificuldades encontradas para se inserir em atividades de pesquisas e/ou extensão estão relacionadas à ocupação; de acordo com o estudo, $71,41 \%$ trabalhavam. Outras dificuldades estão relacionadas a acadêmicos que residem em cidades vizinhas e todos os dias viajam somente para estudar e retornam após as aulas. Esse fator indicou impasse para participar das reuniões de extensão e de pesquisa, pois, além da falta de tempo, os horários dificultavam a integração em ações adotadas pela faculdade, embora todos tenham vivenciado alguma experiência durante a graduação.

Muitos acadêmicos, durante a entrevista, demonstraram dificuldades em participar ou acessar as atividades, questionaram a pouca divulgação durante a graduação ou que os horários para reuniões não atendem à demanda, uma vez que eles dependem de ônibus para se deslocar de outra cidade ou trabalham nos horários estabelecidos, fazendo com que não tenham oportunidade de se integrarem ao ensino, à pesquisa e à extensão.

Para Neves DS e Malta SCL (2014), existem algumas divergências em relação às possíveis dificuldades encontradas pelos alunos, que podem estar relacionadas à dificuldade de articulação do ensino, da pesquisa e da extensão na faculdade por parte dos professores.

Para que haja uma efetiva conexão entre esses pilares, é necessário que o profissional ao qual foi atribuída essa missão busque um método científico baseado em evidências que possam ser aplicadas na prática cotidiana do aluno, através das atividades extracurriculares, como projetos, integrações e inserção da pesquisa desde o início da graduação de modo que seja desenvolvida uma visão crítica e a prática baseadas em evidências, como evidenciado em alguns discursos.

A extensão aparece como uma dimensão essencial para as finalidades das faculdades, tornando-se integrada ao ensino e à pesquisa, estimulando a atuação nas diferentes comunidades e na sociedadeOs professores engajados em tal prática devem ter domínio absoluto desse tripé. É de suma importância e de interesse da faculdade que haja participação de todos os envolvidos, fortalecendo, assim, a comunidade acadêmica e prestando serviço de qualidade à comunidade (FIGUEIREDO WPS, et al.,2016).

Pesquisas científicas e metodologias de ensino são importantes quanto ao desenvolvimento do conhecimento científico na execução de ações de extensão (FIGUEIREDO et al., 2016).

Dessa forma, a inclusão do acadêmico na comunidade ampliando o conhecimento e empregando seu potencial teórico-prático, para aperfeiçoar e contribuir com a comunidade, além de desenvolver atividades que ligam os três pilares, é de suma importância para a instituição de ensino, que além de tornar-se reconhecida pela efetiva atuação na comunidade, entrega profissionais qualificados e experientes para 0 serviço (RODRIGUES ALL, et al., 2013).

Na presente investigação, foram constatadas algumas limitações metodológicas. Uma delas refere-se ao tamanho da amostra, que, devido à metodologia proposta, é relativamente pequena. Contudo, as falas foram representativas e podem abrir perspectivas para compreender outros contextos e realidades de instituições de Ensino Superior da enfermagem no Brasil, bem como apontar caminhos para pesquisas em outros cursos de graduação superior. A escassez de estudos voltados para compreender a importância do tripé da educação superior no ensino público e privado e as dificuldades de inserção, vivenciadas pelos acadêmicos de Enfermagem, dificultam a discussão dos resultados e mostra a carência de pesquisas nessa área. Percebese, ainda, a necessidade de novas discussões e desenvolvimento de pesquisas científicas que aprofundem e que agreguem novos saberes na área de educação, ensino e formação do profissional de enfermagem em consonância com as novas tendências e discussões das Diretrizes Curriculares da Enfermagem. 


\section{CONSIDERAÇÕES FINAIS}

Os resultados apresentados apontam para a necessidade das instituições de ensino se preocuparem em trazer o acadêmico de forma efetiva e completa aos grandes pilares da educação, para que ele possa se desenvolver em todos os âmbitos essenciais para a sua formação, vencendo, assim, as dificuldades encontradas através de planos de ação resolutivos. Trabalhar com o estudante o ensino aliado à prática acadêmica na comunidade contribuiu para o desenvolvimento de pesquisas científicas e permite a formação de profissionais mais cidadãos corresponsáveis com a sua comunidade, em contextos que futuramente poderá estar inserido.

\section{REFERÊNCIAS}

1. ARAGÃO J. Introdução aos estudos quantitativos utilizados em pesquisas científicas. Revista Práxis, 2011; 3(6):5961.

2. BARDIN L. Análise de Conteúdo. Lisboa, Portugal; Edições 70, LDA, 2009; 229p.

3. BARLEM BGT, et al. Fragilidades, fortalezas e desafios na formação do enfermeiro. Escola Anna Nery Revista de Enfermagem, 2012; 16(2): 347-353.

4. BRASIL. Lei no 9.394, de 20 de dezembro de 1996. Lei de Diretrizes e Bases da Educação Nacional. Brasília, DF, 1996.

5. BRASIL. Ministério da Saúde (MS). Conselho Nacional de Saúde, \& Brasil. Ministério da Saúde (MS). Conselho Nacional de Saúde. (2013). Resolução no 466, de 12 de dezembro de 2012. Diário Oficial da União.

6. BRASIL. Ministério do Trabalho e Emprego (MTE). Classificação Brasileira de Ocupações 3ª ed. Brasília: MTE; 2010.

7. BENTO EJ. Desenvolvimento web com PHP e MySQL.Vila Mariana: Casa do Código, 2013; 220 p.

8. DIVINO AEA, et al. A extensão universitária quebrando barreiras. Cadernos de Graduação - Ciências Humanas e Sociais, 2013; 1(16): 135-140.

9. EDUARDO EAA. estrutura organizacional de serviços de enfermagem em hospitais de ensino. 2016. Tese (Doutorado em Enfermagem) -Programa de Pós-Graduação de Enfermagem da Universidade Federal do Paraná, Curitiba, 2016; 185p.

10. FASA. Institucional. Disponível em: http://institucional.santoagostinho.edu.br/index.php?option=com_content\&view=article\&id=147\&ltemid=609

11. FERNANDES MC, et al. Universidade e a extensão universitária: a visão dos moradores das comunidades circunvizinhas. Educação em Revista, 2012; 28(4): 169-179.

12. FIGUEIREDO WPS. et al., Ações de Pesquisa e extensão e Atitudes Científicas de Estudantes da Área de Saúde. Arquivos de Ciência e Saúde,2016; 23(1);47-51.

13. FONTANELLA BJB, et al. Amostragem por saturação em pesquisas qualitativas em saúde: contribuições teóricas. Caderno de Saúde Pública, 2008; 24(1): 17-27.

14. Instituto Brasileiro de Geografia e Estatística (IBGE). Censo 2010. Disponível em: www.ibge.gov.br

15. MINAYO MCS. Análise qualitativa: teoria, passos e fidedignidade. Ciências Saúde Coletiva,2012; 17(3): $621-626$.

16. NEVES DS, MALTA SCL. Ensino, Pesquisa e Extensão: Existem dificuldades Docentes no Ensino Superior para uma integração?. FORMARE, 2014;2(1): 2-12.

17. NOGUEIRA MDP. Manual sobre a extensão universitária para o aluno da UFMG. Belo Horizonte: UFMG; 2005;126p.

18. PIVETTA HMF, et al. Ensino, Pesquisa e Extensão Universitária: Em Busca de uma Integração Efetiva. Linhas Críticas,2010;16(31): 377-390.

19. RODRIGUES ALL, et al., Contribuições da Extensão Universitária na Sociedade, Cadernos de Graduação Ciências Humanas e Sociais,2013; 1(6): 141-148.

20. SIQUEIRA SMC, et al. Atividades extensionistas, promoção da saúde e desenvolvimento sustentável: experiência de um grupo de pesquisa em enfermagem. Escola Anna Nery Revista de Enfermagem,2017;21(1):1-7.

21. SILVA MS. Criando Sites com HTML Sites de Alta Qualidade com HTML e CSS. São Paulo: Novatec, $2008 ; 432$ p.

22. SILVA VO, SANTANA PMMA. Conteúdos curriculares e o Sistema Único de Saúde (SUS): categorias analíticas, lacunas e desafios. Interface-Comunicação, Saúde, Educação, 2014; 19(1): 121-132. 